\title{
EXPLORING STUDENTS CULTURAL EXPERIENCES IN AN INTERNATIONAL LEARNING PROJECT USING DIGITAL REALITY - TALKTECH
}

\author{
Diana Andone, Politehnica University of Timisoara, Romania, Mark Frydenberg, Bentley \\ University, United States of America
}

\section{Introduction}

Digital reality (augmented and virtual reality and other immersive multimedia experiences) have brought about new forms of engagement in a variety of industries in recent years as advances in mobile technologies have raised awareness and usage of these technologies. VR provides a computer-generated simulation of a three-dimensional environment, which seems very real to the person experiencing it. By wearing a special headset or goggles, the user achieves a state of awareness of being present in that virtual environment. Some headsets such as those manufactured by Microsoft, HTC and Oculus offer high-resolution displays to provide immersive experiences for their users. Inexpensive VR viewers require users to insert their smartphones into the viewers to experience VR content.

In an immersive learning environment, "the learner is promoted from an observer of the synthetic world to one of its inhabitants. Immersion promises learners new access to experience." (Jacobson, 2018). The use of augmented and virtual reality technologies has increased the popularity and accessibility of immersive learning environments across the curriculum.

Stewart and Dansie conclude that "immersive media is the future of teaching" (Stewart \& Dansie, 2018) including AR and VR among other new media technologies. Immersive technologies enable students to experience effective intercultural collaboration. Computerbased video and serious games have been found to help learners acquire cultural proficiency. (Fulmore, 2015; Johnson, 2009) As universities continue to incorporate themes of globalization in their curricula, the ability to collaborate and communicate internationally becomes increasingly relevant.

This paper describes the results of TalkTech 2018(TalkTech: An Exploration of Technology, Digital Media, and Culture across Continents, 2019), a learning collaborative study, which since 2008, has paired students at universities in the United State and in Romania, to research and discuss current technologies. The earliest TalkTech projects required students to use what then, were new online tools for collaboration and communication; recordings of those conversations were the deliverables for the project. As student familiarity with online collaboration and communication tools (and the tools, themselves, evolved) the project 


\section{Exploring Students Cultural Experiences in an International Learning Project Using Digital Reality - TalkTech \\ Diana Andone, Mark Frydenberg}

became more complex. Now the tools (often Skype, Facebook Messenger, Google docs and email) that facilitate and enable collaboration and communication in the global economy enable students today to complete projects and create deliverables that are much more complex than what their peers might have produced in earlier years of the TalkTech project. Since 2017, the students have applied their knowledge and digital skills to create and share digital reality content (Andone \& Frydenberg, 2019; Frydenberg \& Andone, 2018b; 2018a) that conveys cultural landmarks in each other's home countries. Students must choose appropriate tools to communicate with their partners and manage the project.

\section{TalkTech 2018}

Globalization means that we will be increasingly connecting with diverse individuals even if we stay at home (Yook \& Turner, 2018; p.380). The TalkTech project adds a global perspective to the digital and information literacy curriculum by pairing students Bentley University enrolled in IT 101, introduction to information technology, with fourth-year students at University Politehnica of Timisoara (UPT) enrolled in a Technology of Multimedia course to explore uses of virtual reality. Each team determines how to communicate using web-based collaboration and communication tools, as they create VR experiences for their partners that present locations of cultural interest in their home cities. Locations for TalkTech 2018 included bus or train stations, places of worship, coffee shops, museum exhibits, historic buildings, outdoor markets, public squares, historical statues, sporting venues, and retail establishments. Students navigate the challenges of no in-person meetings and significant time differences as they. work together for 8 weeks during the Fall 2018 semester to complete the project. At the end of the semester, students share their VR scenes with their partners, and then discuss cultural similarities and differences during a video call.

Rather than using readily available VR content, students create their own immersive learning environments for their international partners, developing their own VR scenes using called CoSpaces EDU (cospaces.edu). The process of researching a cultural landmark, creating and communicating that experience in a VR scene, consuming similar content created by international partners, discussing similarities and differences requires students to interact globally as they them to interact with and fuse digital content, images, and information into a tangible product that provides evidence of their learning and proclivity with digital reality technologies Team members write blog posts in which they reflect on their own experiences as members of an international team, and how they and their group worked through problems and challenges. Students also created Augmented Reality (AR) content to share information about their landmarks and locations using a variety of AR apps such as ROAR, Blippar, and HP Reveal. The TalkTech project requires students to apply their digital skills as they become creators of deeply engaging digital reality content.

To evaluate the students' work for a course grade, both instructors graded each group's project separately. They considered technologies used, evidence of documentation of the 
group's process, and creativity and complexity of the VR scenes created. The instructors shared their evaluations with each other, and then determined final grades for their own students. Each instructor made a virtual appearance by Skype to introduce the project at the beginning, and to debrief with students at the end of the project. Due to a coincidence in class meeting times and the time zone difference, two groups were able to present their projects together over Skype to a joined classroom of both American and Romanian students.

Students from both universities had previous experience using the web, collaboration tools, and mobile devices. The Romanian students were mostly in the age group of 21-23, while the American students were all aged 17-20. Recognizing differences in age, academic interests and backgrounds, and technical abilities, the authors created a project that would be accessible and meaningful to this diverse student population and see these differences as representative of those found in today's global workforce.

The authors studied how collaboratively creating and sharing VR artefacts enables students to better appreciate the possibilities for VR in many settings and encourages the development of new digital and computational thinking skills. Students, in the process, have a glimpse of the culture of their international partners.

\section{The CoSpaces EDU Platform}

When designing a VR immersive experience with CoSpaces EDU, students incorporate threedimensional objects from the platform's library, such as avatars, everyday objects (such as trees, animals, tables and chairs), and background scenes. They personalize the environment by uploading a 360-degree photo created with a 360-degree camera or smartphone app. Students turn their digital images into digital stories by adding animations and interactivity using a visual programming language called CoBlocks. After designing, developing, and coding their virtual worlds with the CoSpaces web application, students can explore their virtual worlds on their mobile devices using the CoSpaces EDU mobile app, available for Android and iOS devices. Through the mobile app, students can visit their virtual worlds by placing their smartphones into a Google Cardboard headset or similar VR viewer, creating a fully immersive experience. They share their VR worlds in blogposts or webpages by providing a link or a QR code.

As an example, one group created a VR experience comparing Starbucks coffee shops in Boston and Timisoara. Clicking an animated avatar in a 360-degree photo outside of the store (Figure 1a) transports the visitor inside the shop, where they can witness a dialogue between two customers discussing their beverage choices and spin the scene around to see the décor and environment of the shop. Clicking the Christmas Tree avatar in the Boston shop (Figure 1b) teleports the viewer to the Timisoara shop, and clicking the Starbucks logo in the Timisoara shop returns the visitor to Boston, creating an almost seamless experience. Figure 1 shows how the VR experience appears in a browser, and on a mobile device to be inserted into a Google Cardboard VR viewer. 
(a)

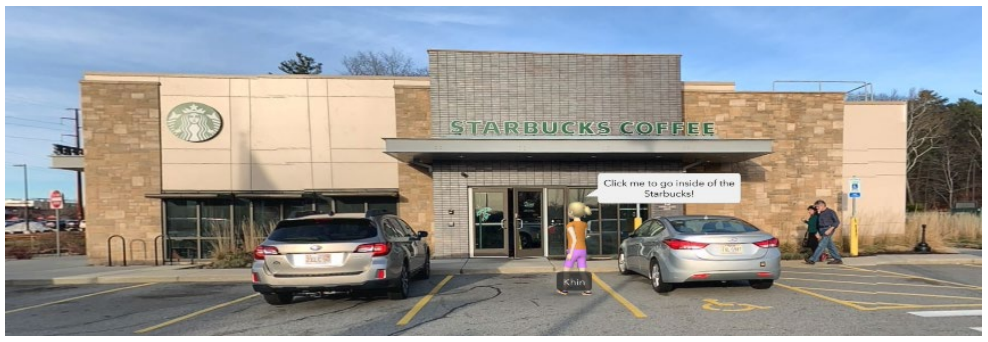

(b)

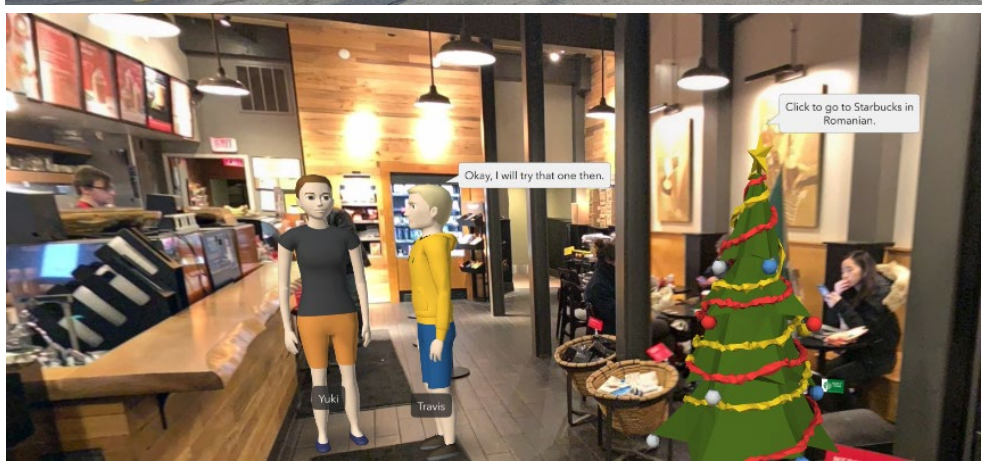

Figure 1. Virtual visit to Starbucks in Boston

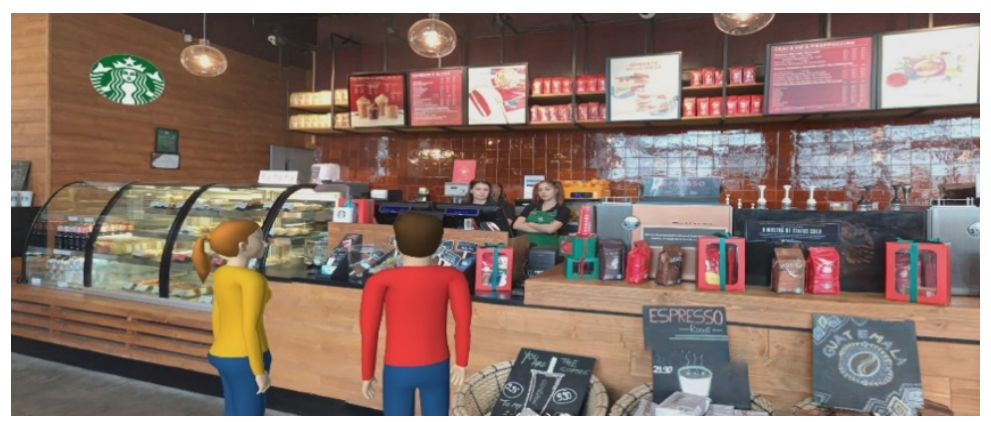

Figure 2. Virtual Visit to Starbucks in Timisoara

Students use the CoBlocks visual programming tool (Figure 3) within the CoSpaces app to give realistic actions such as walking, turning, or gesturing to their avatars, and display conversation bubbles to create a dialogue between them. Simple Turtle Geometry commands carry out the animations. Advanced students can use JavaScript to interact with the avatars and create their scenes. 


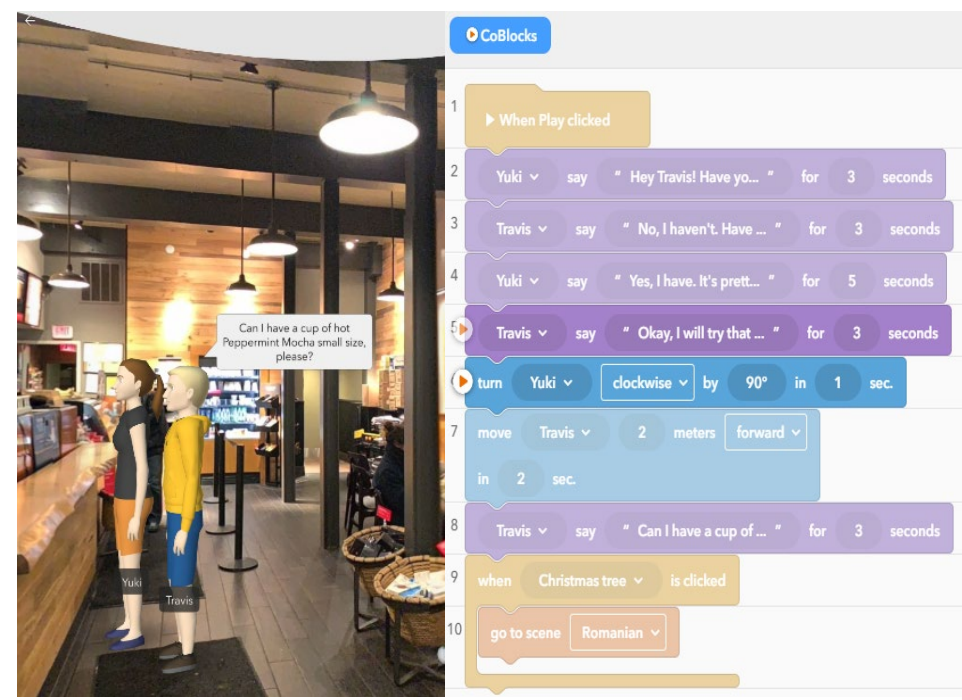

Figure 3. Creating and Animating a VR Scene using CoBlocks

\section{TalkTech 2018 Survey Results}

During the fall 2018 semester, 30 Bentley and 31 UPT students participated in the project. All had prior experience with using mobile phones, text messages, search engines, and online chat tools. Students from both countries cited Facebook messenger as the most popular tool of choice for their group communications, with email the second most popular method of communication. Most groups spoke live at least twice during the project, relying on email, text, and mobile communications to arrange next steps of the project.

Even though students were challenged by using new apps and tools and did not always have the tech skills needed to apply them, all students completed the project successfully. They relied on their team members, the Internet, and their instructors for additional information and guidance when necessary.

\section{Familiarity with VR}

Figure 4 shows that most students were not familiar with tools for creating or consuming immersive content prior to this project. None had used CoSpaces EDU prior to this project. Bentley students, most of whom had no prior coding experience, were offered a one-hour optional training session by a former TalkTech project participant, to learn basic techniques to set up animations using CoBlocks. The UPT students, most of whom had prior coding experience, learned to use CoSpaces on their own.

Several students, when asked about the most important skill learned from a technology perspective, commented, that they "learned how to create" digital reality environments. The experience showed the importance of coding

"I learned that it is crucial to understand basic coding languages and functions in order to be able to apply it to create a VR experience."

and that tools for developing such experiences are evolving. 
"From a technology point of view, I learned more about AR and VR and that it really isn't terrible to create either platform. I also learned that the technology is still in development, and the viewers can be very touchy and unresponsive."

Students also shared sentiments that

"it is simple nowadays to create AR or VR using the appropriate tools."

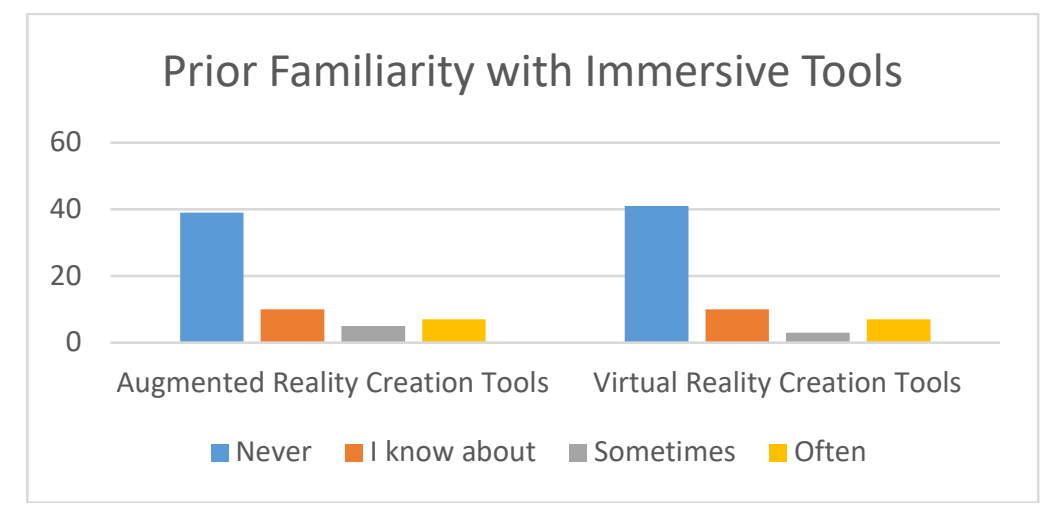

Figure 4. Prior Familiarity with Immersive Media Creation Tools

Said one student,

"This project was really eye opening to the power and convenience behind the rising technologies of virtual reality and augmented reality. It was, to an extent, a learning curve to do some of the aspects of this project. Personally, I never really used AR or VR except for Pokémon Go when it was popular and never would have thought that I would need to create anything similar. Creating the VR was interesting because on the CoSpaces application it was unlike an interface I have used before. It was not difficult nor confusing, it was just different in that it was a three-dimensional space we had to interact with and manipulate. This shows using VR is more suitable for creating more of an experience of an entire space to be more interactive and more exploratory."

\section{Cultural Awareness through Shared VR Experiences}

When asked, "how did this project help you experience the culture of your international partners, and did AR or VR have an impact on this?" American students said:

"This project allowed us to talk with others from completely different cultures. The VR made us feel like would it would be like it was in Romania."

"The AR and VR helped reveal the architecture of Romania and how it is less commercial in layout." 
"The VR definitely impacted my experience of the culture of my international partners because it showed me the similarities and differences that existed as well as how their history impacted the creation of their chosen place".

"The VR allowed us to see an important public square in Romania as though we were actually there."

Romanian students remarked:

"I got an idea about the way they live there. The VR helped a lot in this sense."

"The VR gave us a snapshot of their culture and had a bigger impact for me.

We were able to see what a local marketplace looks like for them and were able to see that things are not extremely different between the US and Romania."

Students from both universities cited difficulties in communication as a reason for not learning about each other's culture. One remarked,

"The VR certainly helped me [see places in Romania], but I didn't think I truly experienced their culture."

Another said,

"We just got to see some places from their place through AR and VR. There was not an impact about their culture."

Many students noted the similarities between cultural landmarks in both countries as a result of viewing each other's immersive experiences.

Of the 30 responses from students at each school, the majority from Romanian students (23/30) were positive about the use of VR on understanding culture of their American partners, and 16/30 responses from American students expressed positive sentiments about using VR to experience the culture of their Romanian partners.

Another student reflected,

"From watching the VR experience we created, I learned that we honestly have everything at our fingertips when it comes to technology. I was able to experience the sights of Timisoara from 4,000 miles away. This added to my learning experience because it felt like what I was doing would be applicable. It wasn't just memorizing data, it was interacting with people across the world, building a space that captured both of our perspectives, and learning how people can think about the same concept in many ways." 


\section{Effective Tools}

Students from both universities considered the tools that were most effective for them to learn about the culture of their partners. They evaluated their learning through real time voice or video conversations, and by viewing or watching a video of the augmented and virtual reality scenes their partners created (see Figure 5.).

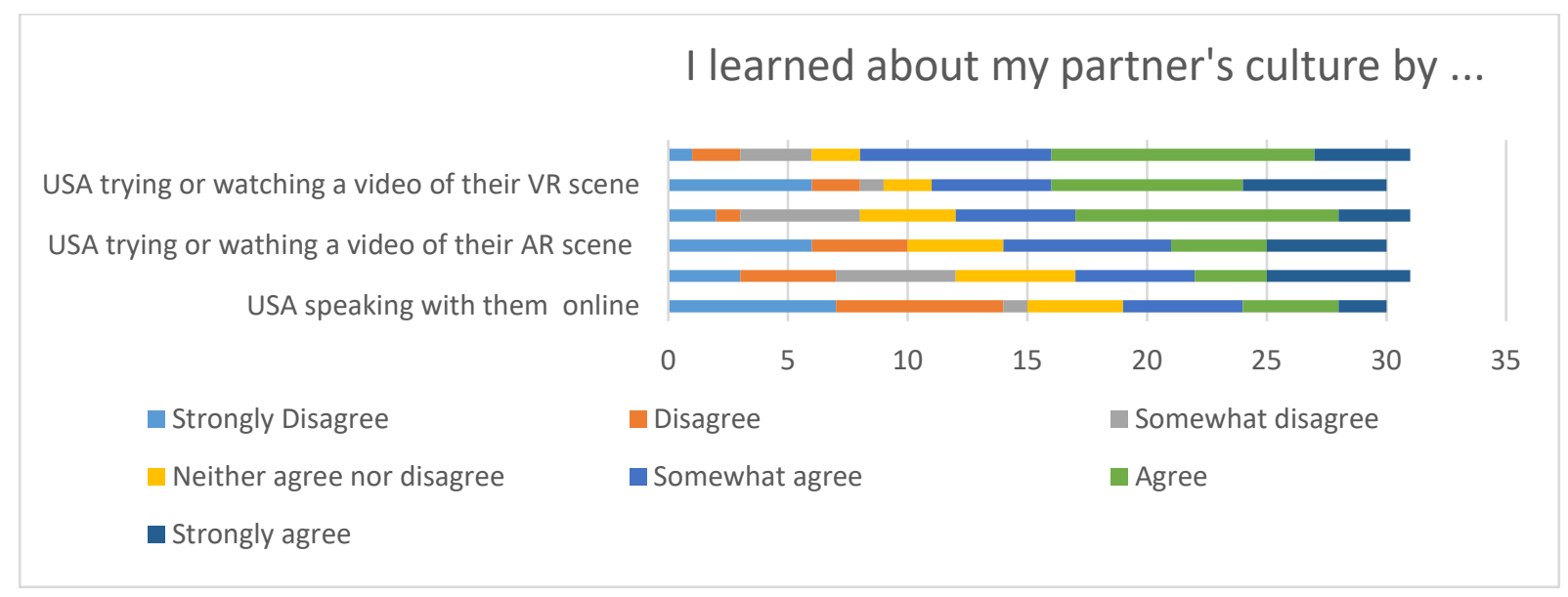

Figure 5. How Students Learned Most About Their International Partner's Culture

Results show that for both the American and Romanian students, both groups agreed or strongly agreed that viewing their partners' VR scenes on their laptops or in a cardboard headset was, the most effective way for them to experience their partner's culture. AR was next, and real time conversations scored the least favourable. This could be because most real time conversations were spent planning the project rather than discussing cultural differences or similarities.

\section{Summary and Conclusions}

The TalkTech 2018 project presents a learning scenario where students interact as members of international teams to create and exchange VR scenes that share aspects of local culture and landmarks. Most students agree that their participation in the TalkTech project taught them about culture, collaboration, working on international teams, and the tools to carry out these tasks. They saw the promise of digital reality technologies in education and in business.

Students also learned that creating, sharing, and exploring VR scenes can change how they experience virtual worlds, as well as how they apply their knowledge gained in those virtual worlds, to the real world. By creating, sharing, and experiencing immersive VR scenes, results suggest that student's use of VR has a favourable impact on their understanding and appreciation of the culture of their international partners.

\section{References}

Andone, D., \& Frydenberg, M. (2019). Creating Virtual reality in a Business and Technology Educational Context. In T. M. tom Dieck, \& T. Jung (Eds.), Augmented Reality and Virtual Reality (pp. 147-159). Switzerland: Springer International Publishing. 
Frydenberg, M., \& Andone, D. (2018a, June 25). Creating Virtual Reality Artefacts to Enhance and Transform Digital Learning. Proceedings of the EdMedia + Innovate Learning, Jun 25, 2018 in Amsterdam, Netherlands, 1543-1552. Retrieved from https://www.learntechlib.org/primary/p/184376/

Frydenberg, M., \& Andone, D. (2018b). Enhancing and Transforming Global Learning Communities with Augmented Reality. Journal of Information Systems Education, 29(1), 37.

Fulmore, Y. (2015). Video Games and the Customization of Learning: Interactive Narratives as a Promising Design Framework for Crafting Inclusive Educational Environments. Proceedings of the Third Conference on GenderIT - GenderIT '15, 32-39. https://doi.org/10.1145/2807565.2807710

Jacobson, D. (2018, April). 12 Steps Toward Immersive Learning. eLearn Magazine. Retrieved February 15, 2019, from http://elearnmag.acm.org/archive.cfm?aid=3152720

Johnson, W. L. (2009). Developing intercultural competence through videogames. Proceeding of the 2009 International Workshop on Intercultural Collaboration - IWIC '09, 99-100. https://doi.org/10.1145/1499224.1499226

Stewart, D., \& Dansie, T. (2018). VFX to teach religion??: learning from immersive media. Proceedings of the ACM SIGGRAPH 2018 Educator's Forum on - SIGGRAPH '18, Article 7. https://doi.org/10.1145/3215641.3215644

TalkTech (2019). An Exploration of Technology, Digital Media, and Culture across Continents. Retrieved from http://talktechproject.net

Yook, E. L., \& Turner, P. K. (2018). Bringing International Perspectives to the Communication Curriculum in the Age of Globalization. Journal of Intercultural Communication Research, 47(5), 375-381. https://doi.org/10.1080/17475759.2018.1480517 